Rev. Elet. em Gestão, Educação e Tecnologia Ambiental (e-ISSN: 2236-1170)

\title{
A ELASTICIDADE DA DEMANDA DE UNIVERSITÁRIOS POR CARNE BOVINA EM SANTA MARIA, RS
}

\section{THE DEMAND ELASTICIY OF UNDERGRADUATE STUDENTS FOR BEEF INS SANTA MARIA, RS}

\author{
Renata Rojas Guerra ${ }^{1}$, Jackeline Karsten Kirinus ${ }^{2}$, Andréa Cristina Dörr ${ }^{3}$, José Laerte Nörnberg ${ }^{4}$
}

\author{
${ }^{1}$ Universidade Federal de Santa Maria (UFSM) - Aluna de mestrado do Programa de Pós-graduação em Engenharia de \\ Produção - PPGEP. E-mail: renata.rojasg@gmail.com \\ ${ }^{2}$ UFSM - Aluna de doutorado do Programa de Pós-graduação em Ciência e Tecnologia de Alimentos: \\ jackeline.kirinus@gmail.com \\ ${ }^{3}$ UFSM - Profa. Adjunto do Departamento de Ciências Econômicas: andreadoerr@yahoo.com.br \\ ${ }^{4}$ UFSM - Prof. Associado do Departamento de Tecnologia e Ciência dos Alimentos: jlnornberg@gmail.com
}

http://dx.doi.org/10.5902/223611708814

\section{RESUMO}

A carne bovina é um alimento de extrema relevância na dieta dos brasileiros. Neste sentido, o objetivo deste estudo foi considerar a influência dos estudantes na determinação do consumo de carne bovina no município de Santa Maria, Rio Grande do Sul, Brasil; bem como estimar as elasticidades renda e preço para a demanda deste produto através do modelo de regressão do tipo log-linear acrescido de uma variável dummy. Para tanto, foi realizada a coleta de dados primários através de formulários aplicados aos estudantes da Universidade Federal de Santa Maria, Brasil (UFSM) e consumidores em supermercados distribuídos pela cidade. A coleta ocorreu no período de março a abril de 2011. Os resultados confirmaram a existência de divergências entre o comportamento de consumo de ambos estratos populacionais. A taxa de crescimento da quantidade de carne bovina consumida pelos habitantes de Santa Maria é 37,9\% menor para os estudantes do que para o restante da população. Quanto às demais variáveis, foi confirmada a existência de uma relação significativa entre estas, portanto seus coeficientes de elasticidade foram cerca de $-0,33 \%$ para os preços e $0,087 \%$ para a elasticidade-renda. Pode concluir que a população do município de Santa Maria, RS é composta por uma parcela significativa de estudantes e que estes frequentemente possuem um padrão de consumo diferenciado, o qual pode influenciar o comportamento da curva de demanda por alimentos do município.

Palavras-chave: bovinos, consumo de carne, estudantes, elasticidade renda, elasticidade preço

\begin{abstract}
The beef is a food of great relevance in the Brazilian diet. In this sense, the objective of this study was to consider the influence of students in determining the consumption of beef in Santa Maria, Rio Grande do Sul, Brazil, and to estimate income and price elasticities for the demand for this product through the model regression of log-linear plus a dummy variable. To this end, we collected primary data through forms applied to students of Federal University of Santa Maria, Brazil (UFSM) distributed in supermarkets and consumers around the city. Data collection occurred from March to April 2011. The results confirmed the existence of differences between the consumption behavior of both strata. The growth rate of the amount of beef consumed by the inhabitants of Santa Maria is $37.9 \%$ lower for students than for the rest of the population. For other variables, confirmed the existence of a significant relationship between these, so their coefficients of elasticity were about $-0.33 \%$ to $0.087 \%$ and prices for the income elasticity. It can be concluded that the population of the municipality of Santa Maria, RS comprises a significant portion of students and they often have a different consumption pattern, which can influence the behavior of the demand curve for food in the city.
\end{abstract}

Key-words: Bovine, meat consumption, students, income elasticity, price elasticity. 


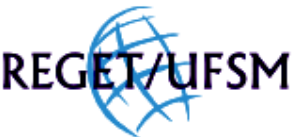

GUERRA et all, v(11), no 11, p. 2479-2492 , JUN, 2013.

Rev. Elet. em Gestão, Educação e Tecnologia Ambiental (e-ISSN: 2236-1170)

\section{INTRODUÇÃO}

No Brasil, a bovinocultura de corte representa grande importância econômica (USDA, 2011). Neste sentido, a carne bovina é um alimento de extrema relevância na dieta dos brasileiros, seja por questões econômicas, culturais ou sociais; este produto é demandado por $99 \%$ da população, fazendo parte da dieta diária de aproximadamente $34 \%$ dos brasileiros, (IBOPE, 2008). Entre os fatores que contribuem para o aumento na produção de carne bovina pode-se citar o crescimento contínuo na demanda doméstica por carne bovina (SILVEIRA et al., 2009; USDA, 2012).

Atualmente, o estado do Rio Grande do Sul ocupa o quinto lugar na atividade pecuária brasileira, com aproximadamente 13,9 milhões de bovinos (MAPA, 2011). Desta forma, o consumo de carne bovina é bastante presente e está fortemente ligado aos fatores naturais (solo, clima) e/ou humanos (tradição, cultura) dos gaúchos (BARCELLOS, 2007). Neste contexto, a análise do comportamento do consumidor de carne bovina é essencial para a compreensão e identificação dos padrões de consumo da população.

Além disso, as mudanças que afetam o consumo de carne bovina na sociedade são constantes e intensas, e acabam por exercer diversas influências sobre o setor produtivo, tendo em vista que são verificadas alterações de conceitos ligados ao consumo de alimentos (ENGEL, 2000; SHETH et al., 2001).

No caso dos estudantes, as mudanças socioeconômicas ocorridas após o ingresso no meio universitário podem contribuir para uma diversificação dos hábitos de consumo. Ademais, "com a inserção na universidade, os jovens oriundos de famílias estruturadas podem sentir dificuldades em prover sua própria alimentação sem a orientação da autoridade parental" afirma Monteiro et al. (2009). Todavia, segundo Blundell (1988) há poucos aspectos da política econômica que não requerem o conhecimento do comportamento do consumidor.

Desta forma, o fato da população da cidade de Santa Maria, RS ser composta por uma parcela significativa de estudantes pode influenciar o comportamento da curva de demanda por alimentos no município. Diante do exposto, o presente estudo visa verificar se existe diferença entre os hábitos de consumo de carne bovina dos estudantes da Universidade Federal de Santa Maria (UFSM) e o restante da população, pretendendo-se encerrar a problemática exposta com o seguinte questionamento: ser estudante, assim como as variáveis renda e preço, influencia a demanda por carne bovina dos consumidores do município de Santa Maria, RS?

Para responder a esta questão foi estimado as elasticidades renda e preço para o consumo de carne bovina na cidade de Santa Maria, RS. Além disso, foi incluída ao modelo uma variável dummy (assume valor igual a 1 se o entrevistado é universitário e 0 caso contrário), a qual permite captar as possíveis diferenças entre o consumo dos estudantes da UFSM com o do restante da população.

\section{METODOLOGIA}




\section{REGEXfUFSM}

Rev. Elet. em Gestão, Educação e Tecnologia Ambiental (e-ISSN: 2236-1170)

Nesta seção serão apresentados os procedimentos necessários à análise empírica acerca da estimação das elasticidades renda e preço para a demanda de carne bovina no município de Santa Maria, RS. Desta forma, além das informações sobre a natureza dos dados, também será demonstrado o modelo econométrico utilizado na análise. Parte-se da estimação de um modelo log-linear de elasticidades acrescido de uma variável de ordem qualitativa cujo intuito é captar a influência do comportamento de consumo dos universitários na demanda total por carne bovina.

\section{Fonte e base de dados}

Para a realização deste trabalho foi utilizada a coleta de dados primários através de formulários aplicados aos consumidores em supermercados distribuídos pela cidade de Santa Maria, RS no período que compreende março e abril de 2011. Desta forma, objetivou-se identificar características de consumo de carne bovina da população em análise através da apuração das seguintes variáveis de interesse: se o entrevistado é estudante universitário ou não; renda familiar mensal em reais; consumo mensal carne bovina em quilogramas; e preço pago por quilograma da carne bovina adquirida.

\section{Amostragem}

A amostragem é uma ferramenta estatística utilizada quando a população - conjunto total dos elementos que se busca analisar - é composta por um número muito grande de elementos, o que impossibilita o acesso a toda a população para a coleta dos dados. Assim, uma amostra é selecionada, com número e característica significativos de elementos dentro da população, para que os resultados da amostra sejam suficientemente informativos e possam inferir conclusões a cerca de parâmetros de toda a população em análise.

Nesta pesquisa, foi utilizada a técnica de amostragem estratificada. Nesta amostragem divide-se a população total a ser inferida em grupos menores, geralmente chamados de estratos, que, segundo Barbetta (2008, p. 48) "são grupos internamente mais homogêneos que a população toda, com respeito às principais variáveis em estudo".

Anteriormente à definição da amostra para a coleta efetiva dos dados, foi realizada uma pesquisa piloto compreendendo um conjunto de 63 consumidores a fim de obter informações sobre a população em análise para determinar o tamanho mínimo da amostra necessária para a pesquisa, visando um nível de confiança de $95 \%$.

Para a amostragem, o cálculo do tamanho da amostra é dado conforme Schneider (2004), através da fórmula (1)

$$
n=\frac{\left(t_{\delta} \frac{\alpha}{2}\right)^{2} \times s^{2} \times N}{e_{0}^{2}(N-1)+\left(t_{\delta}, \frac{\alpha}{2}\right)^{2} \times s^{2}}
$$




\section{REG GUERRA et all, v(11), no 11, p. 2479-2492, JUN, 2013.}

Rev. Elet. em Gestão, Educação e Tecnologia Ambiental (e-ISSN: 2236-1170)

Onde:

$\mathrm{n}$ = tamanho mínimo da amostra calculada;

$t_{\delta} \frac{\alpha}{\alpha},=$ valor de $t_{\text {tab }}$ admitindo ${ }^{\alpha}=5 \%$;

$s^{2}=$ variância obtida através da amostra piloto;

$\mathrm{N}$ = tamanho da população;

$\mathrm{e}_{0}{ }^{2}=$ quadrado do erro amostral, obtido com os dados da amostra piloto

\section{Proporções dos estratos e amostra proporcional}

Segundo dados do Censo de 2000 realizado pelo Instituto Brasileiro de Geografia e Estatística (IBGE, 2000), a população do município de Santa Maria, RS era de 212.410 habitantes. A população está distribuída por bairros, alocados em oito zonas administrativas, as quais serão usadas como estratos para a divisão de nossa amostra. O público foi entrevistado nos supermercados distribuídos pelas zonas correspondentes. A pesquisa piloto, realizada com 63 pessoas, que apresentou um desvio-padrão no consumo de carnes de 1,45 e um erro amostral de 0,183. Assim, amostra calculada para toda a cidade de Santa Maria foi de 264 pessoas.

A amostra total foi distribuída proporcionalmente pelos estratos, conforme a Tabela 1.

Tabela 1. População de Santa Maria, proporções dos estratos e amostra proporcional

\begin{tabular}{llll}
\hline ZONA & POPULAÇÃO & PROPORÇÃO & AMOSTRA \\
\hline Centro Urbano & 61.314 & $29 \%$ & 76 \\
Norte & 27100 & $13 \%$ & 34 \\
Nordeste & 29925 & $14 \%$ & 37 \\
Leste & 15794 & $7 \%$ & 20 \\
Centro-leste & 7543 & $4 \%$ & 9 \\
Sul & 16772 & $8 \%$ & 21 \\
Centro-oeste & 18308 & $9 \%$ & 23
\end{tabular}




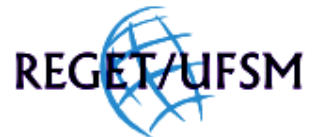

GUERRA et all, v(11), no 11, p. 2479-2492, JUN, 2013.

Rev. Elet. em Gestão, Educação e Tecnologia Ambiental (e-ISSN: 2236-1170)

\begin{tabular}{llll} 
Oeste & 35654 & $17 \%$ & 44 \\
\hline TOTAL & $\mathbf{2 1 2 . 4 1 0}$ & $\mathbf{1 0 0 \%}$ & $\mathbf{2 6 4}$
\end{tabular}

Fonte: Elaborado pelos autores com base nos dados do IBGE (2000) e da Prefeitura Municipal de Santa Maria.

\section{Modelo econométrico}

O método utilizado para a realização do cálculo das elasticidades renda e preço da carne bovina em Santa Maria, RS foi o Ordinary Least Squares (OLS). O uso desta técnica é justificado pela possibilidade de permitir que, através da minimização da soma dos quadrados, sejam encontrados os melhores estimadores lineares não-viesados e de variância mínima.

O modelo a ser estimado consiste em uma regressão do tipo log-linear a partir da qual serão obtidos os coeficientes das elasticidades. Segundo Roppa (2005), a utilização do modelo é explicada pela apresentação das variáveis na escala logarítmica permite que tais coeficientes passem a ser vistos como variações percentuais, o que facilita a interpretação dos mesmos.

Além disso, "deve-se ressaltar que a elasticidade (...) nos informa a variação percentual que ocorrerá em uma variável como reação a um aumento de x\% em outra variável." Conforme Roppa (2005 p. 41-42), a elasticidadade converge com a interpretação obtida a partir da regressão na forma logaritmizada.

No que diz respeito à influência dos estudantes, será introduzida uma variável dummy ao modelo, pois se uma variável qualitativa parece influenciar o regressando, esta claramente deveria ser incluída entre as variáveis explanatórias (GUJARATI \& PORTER, 2011). Desta forma, a equação empregada na análise assume a seguinte especificação:

$$
\operatorname{Ln} C_{\text {came }}=\alpha_{1}+\alpha_{2} D_{1}+\beta_{1} \ln P_{\text {came }}+\beta_{2} \ln Y+\varepsilon_{i}
$$

Em que $L n C_{\text {carne }}$ corresponde ao logaritmo da quantidade em quilograma de carne bovina consumida mensalmente pelas famílias; InP carne é logaritmo do preço pago por quilo de carne bovina adquirida; InY é o logaritmo da renda mensal em reais das famílias santamarienses; $D_{1}$ é a variável dummy dos estudantes do município, assumindo valor igual a 1 se o entrevistado for universitário e 0 caso contrário; e $\varepsilon$ t é o termo de erro estocástico. Os parâmetros $B_{2}$ e 63 representam as elasticidades preço e renda respectivamente, o parâmetro $\alpha_{1}$ é a base do modelo, ou seja, não estudantes, e $\alpha_{2}$ é o parâmetro que indica a influência dos estudantes no consumo de carne bovina em Santa Maria, RS, caso $\alpha_{2}$ seja significativo, entende-se que o padrão de consumo dos universitários difere do restante da população. 


\section{REGEXfHFM}

GUERRA et all, v(11), no 11, p. 2479-2492 , JUN, 2013.

Rev. Elet. em Gestão, Educação e Tecnologia Ambiental (e-ISSN: 2236-1170)

Como o regressor binário é linear, seu coeficiente na verdade representa a semielasticidade, isto é, a variação percentual no regressando em razão de variações unitárias do regressor. Assim, o intercepto dá o logaritmo médio do consumo dos não estudantes e o coeficiente binário a diferença no logaritmo médio do consumo entre ambos os estratos populacionais. Neste caso, para obter a variação percentual das diferenças entre o consumo dos estudantes e dos não estudantes é preciso tomar o antilogaritmo (para a base e) do coeficiente binário estimado, subtrair um dele e multiplicar a diferença por 100. Para o cálculo da regressão e dos testes econométricos foi utilizado o software STATA, versão 11.1 e o Microssoft Excel, versão 2007.

\section{Procedimentos econométricos}

Com o intuito de verificar o grau de eficiência do modelo estimado, bem como realizar uma interpretação dos dados coletados, será realizada a análise estatísticas descritivas das variáveis. Tais análises permitem tanto a identificação do comportamento dos dados quanto à percepção das dimensões de suas variações. Além disso, serão realizados testes de significância global e individual dos parâmetros, bem como diagnósticos de erros de regressão.

Desta forma, através do teste $t$ de Student será possível testar individualmente a hipótese nula de que cada regressor é estatisticamente igual a zero, e, a partir do teste $\mathrm{F}$, verificar a hipótese conjunta de que os verdadeiros regressores parciais são simultaneamente iguais a zero. No que diz respeito ao grau de ajustamento do modelo, será utilizado o coeficiente de determinação $\left(R^{2}\right)$. Este coeficiente permite averiguar a proporção de variação total da variável dependente que é explicada pela variação da variável independente. O valor de $R^{2}$ pode variar entre zero e um $\left(0<R^{2}<1\right)$. Segundo Carvalho \& Bacchi (2007), quanto mais próximo estiver do valor de um, melhor será a qualidade do ajustamento.

Também serão realizadas análises no sentido de verificar se os pressupostos do modelo de regressão linear clássico são atendidos pela metodologia proposta. A destacar o teste de normalidade de Jarque Bera e o teste de Breusch-Pagan-Godfrey (BPG) no propósito de detectar o problema de heterocedasticidade já que "em dados de corte transversal envolvendo unidades heterogêneas, a heterocedasticidade pode ser a regra e não a exceção" (GUJARATI \& PORTER, 2011, p. 381). Ainda segundo o autor, não é necessária a realizar diagnósticos de autocorrelação já que este é um problema característico de modelos de séries temporais.

\section{RESULTADOS E DISCUSSÃO}

Nas Tabelas 2 e 3 estão contidas as estatísticas básicas para todas as variáveis analisadas no presente estudo. Assim, é possível observar os valores das médias; máximo e mínimo; desvio padrão para as variáveis renda; quantidade e preço da carne bovina. 


\section{REGEX}

GUERRA et all, v(11), no 11, p. 2479-2492 , JUN, 2013.

Rev. Elet. em Gestão, Educação e Tecnologia Ambiental (e-ISSN: 2236-1170)

As estatísticas sumárias das variáveis em análise retratam claramente a divergência de comportamento entre os dois estratos populacionais. No caso da renda, destaca-se a magnitude do desvio padrão que, a despeito de apresentar valores de máximo e mínimo bastante similares entre os estratos populacionais, apresentou um grau mais elevado para os estudantes em relação ao restante da população. Este resultado indica que os estudantes de Santa Maria possuem um nível de renda mais heterogêneo que o do restante dos habitantes do município. No que diz respeito às demais variáveis, destacam-se os dados referentes à população de não estudantes que, apesar de ter um intervalo mais amplo entre os valores máximos e mínimos, apresentou um menor desvio padrão.

Tabela 2. Estatísticas básicas das variáveis referentes à população em geral

\begin{tabular}{lllll}
\hline VARIÁVEIS & MÍNIMO & MÁXIMO & MÉDIA & DESVIO PADRÃO \\
\hline Renda & 166 & 11000 & 2072.934 & 1799.909 \\
Preço & 3.4 & 60 & 11.40944 & 5.236315 \\
Quantidade consumida & 1.2 & 48 & 10.90047 & 7.391061 \\
\hline
\end{tabular}

Fonte: Elaborado pelos autores com base nos dados da pesquisa.

Tabela 3. Estatísticas básicas das variáveis referentes aos estudantes

\begin{tabular}{lllll}
\hline VARIÁVEIS & MÍNIMO & MÁXIMO & MÉDIA & DESVIO PADRÃO \\
\hline Renda & 180 & 10000 & 1843.933 & 2134.298 \\
Preço & 3 & 22 & 11.1572 & 4.28906 \\
Quantidade consumida & 0.4 & 32 & 7.632 & 6.117678 \\
\hline
\end{tabular}

Fonte: Elaborado pelos autores com base nos dados da pesquisa.

Nas Figuras 1 e 2 são apresentadas as distribuições de frequência da quantidade de carne bovina consumida entre os dois estratos populacionais, evidenciando assim as divergências entre o comportamento de consumo de ambos os grupos. 


\section{REGETHFSM}

GUERRA et all, v(11), no 11, p. 2479-2492 , JUN, 2013.

Rev. Elet. em Gestão, Educação e Tecnologia Ambiental (e-ISSN: 2236-1170)

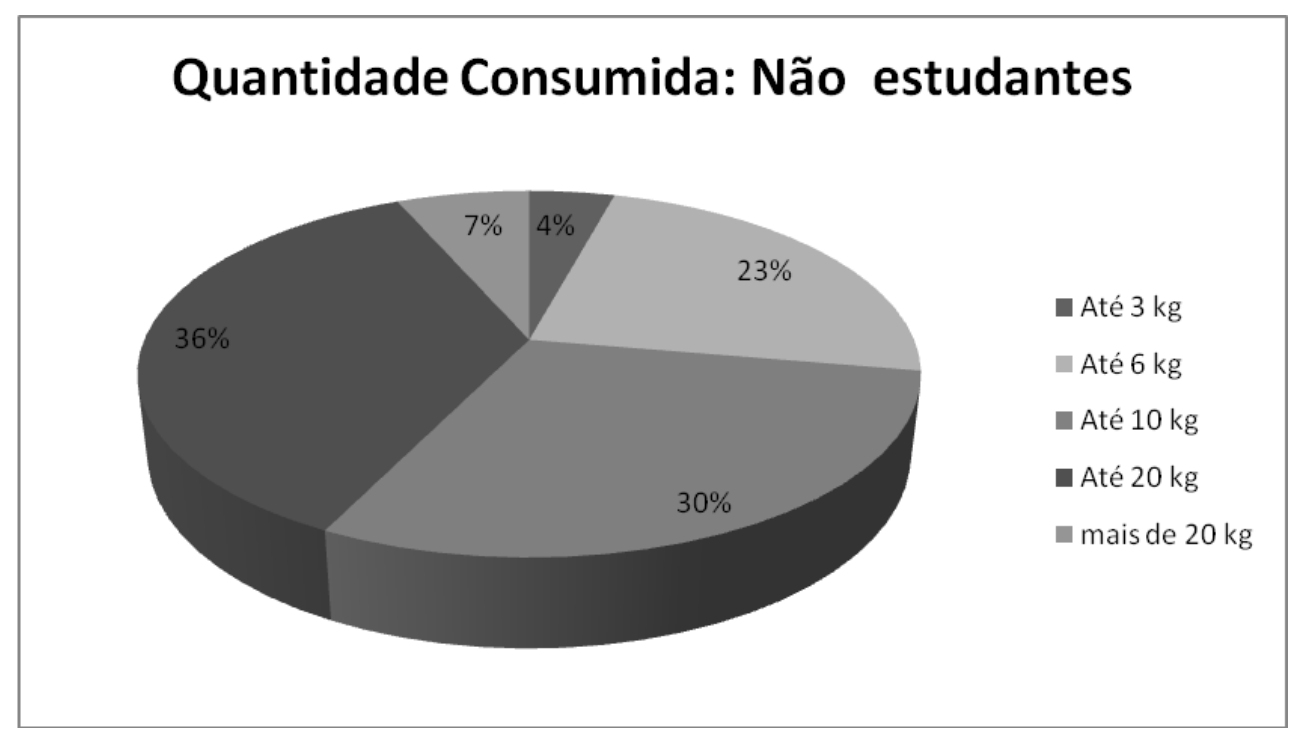

Figura 1. Distribuição de frequência da quantidade de carne bovina consumida pelos não estudantes Fonte: Elaborado pelos autores com base nos dados da pesquisa.

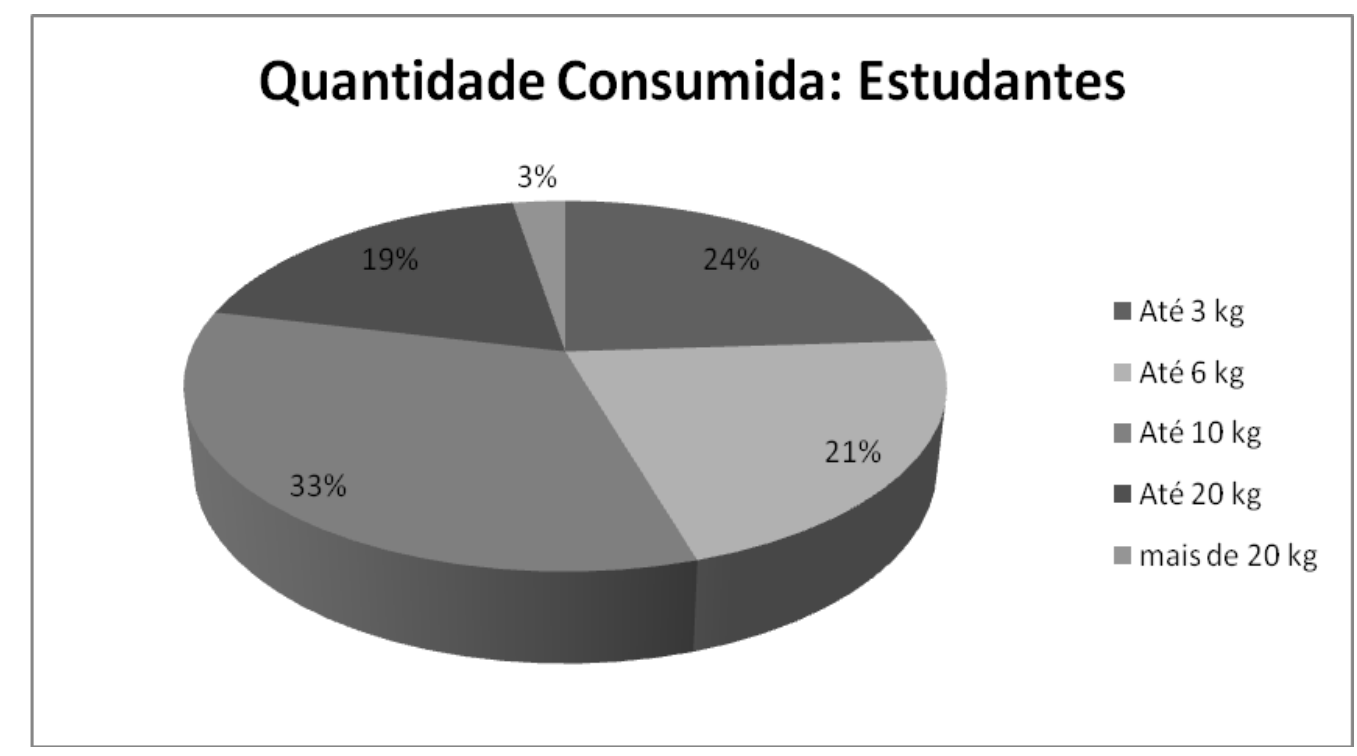

Figura 2. Distribuição de frequência da quantidade de carne bovina consumida pelos estudantes Fonte: Elaborado pelos autores com base nos dados da pesquisa.

Cerca de $45 \%$ dos estudantes consomem até seis quilogramas de carne bovina por mês, enquanto que apenas $27 \%$ do restante da população apresentam este nível de consumo. Nas faixas de consumo superiores, acima de 10 quilogramas, destaca-se a população não estudante, já que 


\section{REGEXJUFS}

Rev. Elet. em Gestão, Educação e Tecnologia Ambiental (e-ISSN: 2236-1170)

$43 \%$ desta têm seu consumo mensal neste patamar, enquanto que apenas $22 \%$ dos estudantes santamarienses consomem mais de 10 quilos de carne bovina por mês. Entretanto, resta verificar se estas evidências são estatisticamente significativas, o que é realizado através do modelo de regressão que considera o consumo dos estudantes como variável dummy (Tabela 4).

Tabela 4. Resultados do modelo de regressão do consumo dos estudantes segundo a variável dummy

\begin{tabular}{|c|c|c|}
\hline VARIÁVEIS & ESTIMATIVA DOS PARÂMETROS & VALORES DE $T[\mathrm{P}]$ \\
\hline Constante & 2.330875 & $5.56[0.000]$ \\
\hline Efeito de ser estudante & -0.476467 & $-4.84[0.000]$ \\
\hline Logaritmo dos preços & -0.3345499 & $-2.92[0.004]$ \\
\hline Logaritmo da renda & 0.0873267 & $1.56[0.119]$ \\
\hline Estatística F( 2, 261) = & $11.64[0.0000]$ & \\
\hline
\end{tabular}

Fonte: Elaborado pelos autores com base nos dados da pesquisa através do software Stata 11.1.

Entretanto, antes de interpretar os resultados demonstrados na Tabela 4, é necessário diagnosticar se houve a violação de algum dos pressupostos do modelo de regressão linear clássico. Assim, para verificar a hipótese de normalidade dos resíduos da regressão estimada, são apresentados o histograma da distribuição dos resíduos, Figura 3, e as estimações dos coeficientes de assimetria, curtose e do teste de Jarque-bera (Tabela 5).

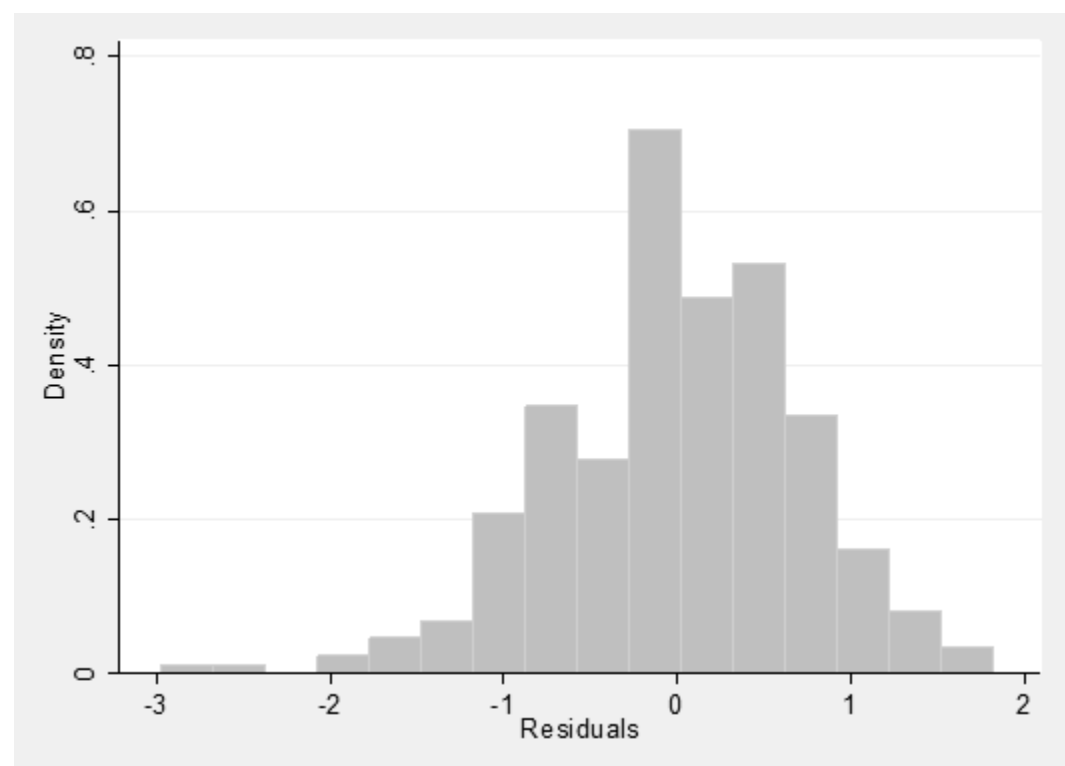

http://cascavel.ufsm.br/revistas/ojs-2.2.2/index.php/reget 


\section{REGEIfHFSM}

GUERRA et all, v(11), no 11, p. 2479-2492, JUN, 2013.

Rev. Elet. em Gestão, Educação e Tecnologia Ambiental (e-ISSN: 2236-1170)

Figura 3. Histograma dos resíduos da regressão estimada, onde Density = densidade; Residuals = residual Fonte: Elaborado pelos autores com base nos dados da pesquisa.

O diagrama apresentado na Figura 3 demonstra que é provável que os resíduos não tenham uma distribuição normal perfeita, já que grande parte das observações concentra-se na extremidade à direita do eixo. Fato este que é corroborado tanto pelos valores dos coeficientes de assimetria $(-0,4663)$ e curtose $(3,90)$, que no caso da distribuição normal devem estar próximos de zero e três respectivamente, quanto pelo teste de normalidade de Jarque-bera, o qual apresentou um valor calculado muito elevado $(20,18)$, permitindo a rejeição da hipótese nula de normalidade.

Entretanto, conforme Gujarati \& Porter (2011), esta hipótese não é essencial se o objetivo for apenas estimação. Além disso, de acordo com o teorema do limite central, mesmo se os termos de erro não forem normalmente distribuídos, os estimadores OLS serão em amostras assintóticas.

Tabela 5. Coeficientes de assimetria e curtose e resultados do teste de Jarque-Bera para o resíduo da regressão

\begin{tabular}{ll}
\hline TESTES & COEFICIENTE \\
\hline Assimetria & $-0,4663069$ \\
Curtose & 3,901179 \\
Jarque Bera & $20,18\left[4.1 \mathrm{e}-05^{*}\right]$ \\
Breusch-Pagan-Godfrey & $10,30\left[0,0013^{*}\right]$ \\
\hline
\end{tabular}

Fonte: Elaborado pelos autores com base nos dados da pesquisa através do software Stata 11.1. Legenda: * probabilidade de aceitar a hipótese nula

Quanto ao problema da multicolinearidade, foi verificado que este não parece estar presente no modelo de parâmetros significativos, tanto individualmente, quanto em conjunto. Todavia, ainda é preciso testar se o modelo é homocedástico, pois na presença da heterocedasticidade a variância do coeficiente estimado por OLS é um estimador viesado e, em consequência, os testes t e $\mathrm{F}$ usados habitualmente deixam de ser confiáveis.

Conforme ressaltado na sessão 2.3, o teste utilizado neste estudo para detectar o problema de heterocedasticidade é o BPG, o qual considera a variância constante como hipótese nula. No modelo em questão, o valor calculado do teste BPG $(10,30)$ é bastante alto, permitindo a rejeição da hipótese nula de que o modelo é homocedástico, neste caso medidas corretivas são necessárias. Um do mais usuais métodos de correção deste problema são os erros padrão corrigidos para heterocedasticidade de White, também conhecidos como erros padrão robustos. 


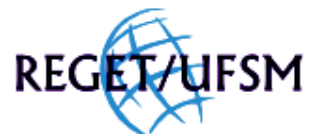

GUERRA et all, v(11), no 11, p. 2479-2492 , JUN, 2013.

Rev. Elet. em Gestão, Educação e Tecnologia Ambiental (e-ISSN: 2236-1170)

Estes permitem que a estimativa seja realizada de modo que as estatísticas válidas assintoticamente possam ser feitas sobre os verdadeiros valores dos parâmetros (WHITE, 1980).

Desta forma, os valores dos coeficientes estimados por OLS permanecem os mesmos, porém os erros padrão podem ser maiores ou menores que os não corrigidos, dado o simples diagnóstico do problema não permite determinar se o viés é positivo (superestimativa) ou negativo (subestimativa).

Tabela 6. Resultados da regressão corrigida pelo procedimento dos erros padrão robustos de White

\begin{tabular}{|c|c|c|}
\hline VARIÁVEIS & ESTIMATIVA DOS PARÂMETROS & VALORES DE $T[P]$ \\
\hline Constante & 2.330875 & $5.56[0.000]$ \\
\hline Efeito de ser estudante & -0.476467 & $-4.84[0.000]$ \\
\hline Logaritmo dos preços & -0.3345499 & $-2.92[0.023]$ \\
\hline Logaritmo da renda & 0.0873267 & $1.56[0.149]$ \\
\hline Estat. $F(2,261)=9.48$ & {$[0.0000] \quad R^{2}=0.1095$} & \\
\hline
\end{tabular}

Fonte: Elaborado pelos autores com base nos dados da pesquisa através do software Stata 11.1.

Tendo por base os resultados de ambos as regressões (Tabelas 4 e 6), é possível constatar que os resultados do teste $\mathrm{F}$ corroboram a significância global da regressão estimada tanto no modelo inicial quanto no corrigido, ou seja, revelam que todas as variáveis são significativas em conjunto.

Quanto à significância individual dos parâmetros observa-se que tanto a constante quanto os preços e a variável dummy para o comportamento são significativos a $1 \%$ no modelo inicial, entretanto, no modelo corrigido os preços passam a ser significativos apenas ao nível de $5 \%$. Já a variável renda familiar, que só poderia ser considerada a níveis de significância superiores a 11,9\% no modelo inicial, no modelo corrigido ela se torna ainda menos significante, podendo ser considerada apenas ao nível de 14,9\%. No que diz respeito à estatística do coeficiente de determinação $\left(R^{2}\right)$, os dados mostram que grau de explicabilidade das variáveis independentes sobre o consumo de carne bovina em Santa Maria está em torno de 10,95\%, valor que supera tanto o encontrado no modelo não corrigido $(10,01 \%)$ quanto os $4,59 \%$ encontrados no modelo que não considera o consumo dos estudantes como variável dummy..

Como os parâmetros são significativos, faz-se possível a interpretação das estimações no sentido de que estes representam os coeficientes de elasticidade da demanda de carne bovina para o município de Santa Maria, RS. Ademais, fica atestada a hipótese de que os estudantes santamarienses possuem um padrão de consumo de carne bovina divergente do restante da 


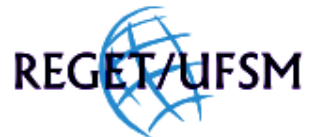

GUERRA et all, v(11), no 11, p. 2479-2492 , JUN, 2013.

Rev. Elet. em Gestão, Educação e Tecnologia Ambiental (e-ISSN: 2236-1170)

população, já que o parâmentro $\alpha_{2}$ é significativo. Assim, aplicando-se a transformação do antilogaritmo do coeficiente estimado para a variável binária é possível constatar que, a taxa de crescimento da quantidade de carne bovina consumida pelos habitantes de Santa Maria será $37,9 \%$ menor para os estudantes do que para o restante da população. Este resultado pode ser justificado pelo fato de que, conforme Borges \& Lima (2004), na maioria dos casos os estudantes tendem a realizar suas refeições fora de casa e em horários não padronizados.

No que diz respeito aos coeficientes de elasticidade, a elasticidade-preço indica que aumentos de $1 \%$ no preço da carne tendem a ocasionar diminuições de aproximadamente $0,33 \%$ em seu consumo. Este resultado converge com a teoria econômica que pressupõe que aumentos nos preços, coeteris paribus, afetam negativamente o consumo. Quanto à elasticidade-renda, uma variação de $1 \%$ na renda das famílias de Santa Maria, RS, tende a ocasionar uma variação positiva de cerca de $0,087 \%$ no consumo de carne, o que também converge com a teoria econômica, no entanto ressalta-se que este coeficiente só é significativo ao nível de 14,9\%.

Ao comparar os resultados do presente estudo com os obtidos por Carvalho \& Bacchi (2007), os quais calcularam a elasticidade-renda referente à demanda de carne bovina, suína e de frango para diversos estados do Brasil, dentre as quais se destaca o estado do Rio Grande do Sul, é possível perceber que os resultados encontrados pelos autores para a região divergem com os da presente análise. A divergência se dá no que concerne à magnitude dos coeficientes de elasticidade-renda média para a carne de primeira, já que esta ficou em torno dos 0,74\%. Entretanto, ao se tratar da elasticidade-renda para a carne de segunda obteve-se um valor mais próximo ao encontrado, cerca de $0,18 \%$.

Além disso, cabe destacar que neste trabalho os estratos populacionais foram os estudantes universitários e o restante da população. Ademais, é importante atentar para o fato de que há uma distinção entre o período abordado pelos autores e o utilizado no presente estudo, o que compromete a eficiência de tais comparações.

\section{CONCLUSÕES}

Faz-se necessário identificar os padrões de consumo de carne bovina, seja devido à representatividade deste produto na cesta de consumo alimentar da população, ou pela relevância do mercado de carne bovina na economia do país. Neste contexto, ao destacar que a população do município de Santa Maria, RS é composta por uma parcela significativa de estudantes e que estes frequentemente possuem um padrão de consumo diferenciado, pode-se evidenciar uma influência no comportamento da curva de demanda por alimentos do município.

Também, conforme a análise da distribuição de frequência da quantidade de carne bovina consumida por ambos os estratos populacionais evidenciou-se divergências entre o comportamento de consumo de ambos os grupos; entretanto, ao incorporar no modelo uma variável que capta a influência do consumo dos estudantes santamarienses, é possível corroborar tal hipótese. Desta forma, constatou-se que a taxa de crescimento da quantidade de carne bovina 


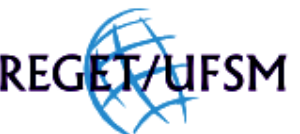

GUERRA et all, v(11), no 11, p. 2479-2492 , JUN, 2013.

Rev. Elet. em Gestão, Educação e Tecnologia Ambiental (e-ISSN: 2236-1170)

consumida pelos habitantes de Santa Maria é $37,90 \%$ menor para os estudantes do que para o restante da população.

Quanto às demais variáveis, foi confirmada a existência de uma relação significativa entre estas. Os coeficientes de elasticidade foram cerca de $-0,33 \%$ para os preços e $0,087 \%$ para a elasticidade e renda. Assim, torna-se evidente a necessidade de promover uma separação entre o público universitário e o restante da população do município, devido aos padrões de comportamento distintos.

\section{REFERÊNCIAS BIBLIOGRÁFICAS}

BARBETTA, P. A. Estatística Aplicada às Ciências Sociais. 7. ed. Florianópolis: Ed. da UFSC, 2008.

BARCELLOS, M. D. de. "Beef-lover": Um estudo cross-cultural sobre o comportamento de consumo de carne bovina. [Tese de Doutorado]. Porto Alegre (RS): Universidade Federal do Rio Grande do Sul; 2007.

BORGES, C. M.; LIMA FILHO, D. de O. Hábitos alimentares dos estudantes universitários: um estudo qualitativo. In: SEMINÁRIOS DE ADMINISTRAÇÃO, São Paulo. Anais..., São Paulo: FEA/USP, 2004.

BLUNDELL, R. Theory and Empirical Evidence - a Survey. The Economic Journal, v. 98, n. 389, p. 16-65, 1988.

CARVALHO, T. B. de.; BACCHI, M. R. P. Estudo da Elasticidade-Renda da Demanda de Carne Bovina, Suína e de Frango no Brasil. XLV Congresso Sociedade Brasileira de Economia, Administração e Sociologia Rural, Londrina - PR, 2007.

ENGEL, J.; BLACKWELL, R.; MINIARD, P. Comportamento do consumidor. 8. ed. Rio de Janeiro: Livros Técnicos e Científicos, 2000.

GUJARATI, D.; PORTER, D. Econometria Básica. 5. Ed. Porto Alegre: AMGH, 2011.

IBGE. Censo Demográfico de 2000. Disponível em:

<http://ibge.gov.br/home/estatistica/populacao/acao/default_censo_2000.shtm>. Acesso em: 03 abr. 2011.

IBOPE. Instituto Brasileiro de Opinião Pública e Estatística. 2008. Disponível em:

<http://www.ibope.com.br/opiniao_publica/downloads/ibope_inteligencia_wwf jun06.pdf>. Acesso em: 17 ago. 2011.

MAPA. MINISTÉRIO DA AGRICULTURA PECUÁRIA E ABASTECIMENTO. Mercado Interno. 2011. Online. Disponível em: <http://www.agricultura.gov.br/animal/mercado-interno>. Acesso em: 15 mai. 2012.

MONTEIRO, M. R. P.; ANDRADE, M. L. de O.; ZANIRATI, V. F.; SILVA, R. R. Hábito e consumo alimentar de estudantes do sexo feminino dos cursos de Nutrição e de Enfermagem de uma universidade pública brasileira. Revista APS, v. 12, n. 3, p. 271-277, 2009.

Prefeitura Municipal de Santa Maria. Mapa da Divisão Urbana de Santa Maria. Disponível em: <http://www.santamaria.rs.gov.br/docs/mapa divisao urbana.pdf>. Acesso em: 03 abr. 2011.

ROPPA, B. F. Evolução do consumo de gasolina no Brasil e suas elasticidades: 1973 a 2003. Rio de Janeiro: UFRJ, 2005. (Monografia de Bacharelado do Instituto de Economia), Universidade Federal do Rio de Janeiro, 2005. 


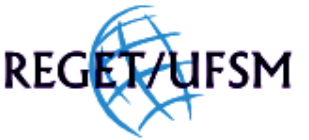

GUERRA et all, v(11), no 11, p. 2479-2492 , JUN, 2013.

Rev. Elet. em Gestão, Educação e Tecnologia Ambiental (e-ISSN: 2236-1170)

SHETH, J.; MITTAL, B.; NEWMAN, B. Comportamento do cliente: indo além do comportamento do consumidor. São Paulo: Atlas, 2001.

SCHNEIDER, L. Estimativa dos Gastos dos Alunos da UFSM (2002): Aplicação da Amostragem Estratificada Proporcional.Santa Maria: UFSM, 2004. (Monografia apresentada ao Curso de Especialização em Estatística e Modelagem Quantitativa), Universidade Federal de Santa Maria, 2004.

SILVEIRA, V. C. P.; VIANA, J. G. A; WERNER JUNIOR, C. Preço pago pelo consumidor de carne bovina nas diferentes regiões econômicas do Rio Grande do Sul, Brasil. Ciência Rural, v. 39, n. 4, p. 1201-1207, 2009.

USDA. UNITED STATES DEPARTMENT OF AGRICULTURE. Livestock and poultry: word markets and trade. Washington: D.C, 2011. Online. Disponível em: <http://www.fas.usda.gov/psdonline/circulars/livestock_poultry.pdf>. Acesso em: 03 mar. de 2012.

USDA. UNITED STATES DEPARTMENT OF AGRICULTURE.. Foreign Agricultural Service: Global Agricultural Information Network. Washington, D.C., 2012. Online. Disponível em:

<http://gain.fas.usda.gov/Recent\%20GAIN\%20Publications/Livestock\%20and\%20Products\%20Semiannual_Brasilia_Brazil_1-3-2012.pdf>. Acesso em: 09 mar. 2012.

WHITE, H. A heteroskedasticity-consistent covariance matrix and a direct test for heteroskedasticity. Econometrica, v. 48, p. 817-838, 1980. 